\title{
Ambulatory Care in IR: Time to Engage
}

\author{
Michael J. Lee ${ }^{1}$ (i) $\cdot$ Christoph A. Binkert ${ }^{2}$
}

Received: 9 March 2021 / Accepted: 11 March 2021/Published online: 21 March 2021

(C) Springer Science+Business Media, LLC, part of Springer Nature and the Cardiovascular and Interventional Radiological Society of Europe (CIRSE) 2021

Healthcare expenditure in Europe is increasing on a yearly basis with the average healthcare expenditure among European nations amounting to $9.9 \%$ of GDP (gross domestic product) in 2018 [1]. Methodologies to reduce cost and use funding more efficiently have become the focus of most healthcare systems. In this regard, the development of day care surgery is increasing throughout the USA and Europe, particularly in the last two decades. Day surgery, in its early days, was reserved for simple procedures in selected patients. However, the pendulum has swung now towards considering day surgery in many elective surgeries. Hip replacement and knee replacement procedures as well as laparoscopic cholecystectomy are now considered as potential day surgery cases. Economically, it has been shown that hospital costs for day surgery versus inpatient surgery are $25 \%-68 \%$ lower [2].

Interventional radiology (IR) is a minimally invasive procedural specialty that does not require the services of anesthesiology for the vast majority of procedures which simplifies the transition to day care IR. IR is, therefore, a specialty that is well suited to ambulatory care because of the above and its low incidence of complications due to its minimal invasive nature. In certain European countries, ambulatory care for IR is well developed. However, there is a wide variation both within and between European countries in terms of the delivery of IR ambulatory care. The advantages of ambulatory care for IR are substantial and include reduction in hospital costs, increase in throughput of patients and a reduction in waiting lists. During the current pandemic, the ability to perform elective day case IR procedures separately from inpatients proved to be beneficial and allowed elective IR procedures to continue.

While IR is ideally suited to ambulatory care procedures, there are barriers to the widespread adoption of ambulatory care throughout Europe. These include: reimbursement issues in that it may be more financially advantageous for hospitals and interventionalists if patients are hospitalized for more than $24 \mathrm{~h}$; national regulations may preclude a shift to ambulatory care; hospital design and lack of day care beds may not be configurable to an IR day care system and lack of home or community support may preclude some patients from participating in ambulatory care. For IR in Europe, one of the most significant factors in many countries is reimbursement differences for the same procedure between an inpatient and day case setup. Procedure reimbursement for physicians must be harmonized to reflect an equal or even higher price paid for day case versus inpatient IR. Such an incentive would automatically increase the number of day care IR which would help to decrease the overall costs without the necessity for additional regulations.

Michael J. Lee

mlee@rcsi.ie

Christoph A. Binkert

christoph.binkert@ksw.ch

1 Department of Radiology, Royal College of Surgeons in Ireland, Beaumont Hospital, Dublin 9, Ireland

2 Radiology and Nuclear Medicine, Kantonsspital Winterthur, Brauerstrasse 15, CH-8401 Winterthur, Switzerland 
Clearly, embracing ambulatory care IR will not happen overnight and a cultural change and change in reimbursement mechanisms will be necessary for many hospitals and healthcare systems. Ambulatory care IR must be adequately resourced to be safe and effective. There will be cost involved in terms of the initial setup of any day case IR facility in that access to an outpatient clinic/pre-assessment area, day care beds, patient pathways and adequate nursing and clerical support are mandatory. Governance and audit will also be important in setting up a day care service. The concept of the IR team and the more advanced role of nursing and clerical colleagues in terms of developing patient pathways, scheduling and pre-assessment and discharge planning are key. Day care beds should also be close to the IR suite to reduce transport times and increase efficiency. CIRSE (Cardiovascular and Interventional Radiology Society of Europe) recently instituted a task force on IR ambulatory care who produced a Webinar on ambulatory care in IR [3] which can be viewed on the CIRSE website. The latter is an introductory webinar on the topic and viewing is recommended.

In summary, elective IR procedures are ideally suited to ambulatory care and as interventional radiologists become more involved in ambulatory care, we believe that in the future, a paradigm shift will occur in that day case IR will be considered the norm for most elective IR procedures.

In the words of Goethe:

Whatever you can do, or dream you can do, begin it. Boldness has genius, power and magic in it.

Funding None.

Compliance with Ethical Standards

Conflict of interest There is no conflict of interest.

Consent for Publication For this type of study consent for publication is not required.

\section{Reference}

1. Healthcare expenditure across the EU. Ec.europa.eu/Eurostat. HLTH_SHA11_HF

2. Mitchell JM, Carey KA. comparison of ambulatory surgery center production costs and Medicare payments: Evidence on colonoscopy and endoscopy. Med Care. 2016;54(2):126132.

3. Webinar 2021 Ambulatory care in IR: the time is now. Library.cirse.org.

Publisher's Note Springer Nature remains neutral with regard to jurisdictional claims in published maps and institutional affiliations. 